\title{
The Association of Clinical Pathologists: 91st general meeting
}

The 91st general meeting was held at Imperial College, London, from 19 to 21 September 1973. Abstracts of the scientific communications follow. The Presidential Address, 'Haematology-trends and opportunities', given by Professor E. OK. Blackburn, and the Dyke Foundation Lecture, 'Men and machines', given by Professor N. H. Martin, are publishedEn the November issue.

Giant Nuclear Masses in the Lungs and Blood in Malignant Malaria

H. B. GOODAll (Royal Infirmary, Dundee) Numerous large naked nuclear masses have been found in the lungs of two patients dead from malignant (subtertian, falciparum) malaria. These structures are probably derived from vascular endothelial cells damaged and shed in the process of disseminated intravascular coagulation which is the pathogenetic mechanism causing the complications of malignant malaria, particularly the cerebral damage. Ten buffy coat smears and 30 thick films of blood from one of these patients taken during the terminal illness all contain nuclear masses. Microscopy of such simple preparations, of quantitative modifications of them, or of more elaborate cell concentrates, appears to offer a new method for the detection of disseminated intravascular coagulation in malaria, a method to supplement current diagnostic tests, eg, the estimation of fibrin degradation products.

Clinical and Diagnostic Importance of Synovial Fluid Examination

B. VERNON-ROBERTS (The London Hospital Medical College, London) The examination of a sample of synovial fluid from a joint effusion can establish or exclude the diagnosis of crystal synovitis or septic arthritis; it can often provide sufficient evidence for a definite diagnosis in effusions of uncertain aetiology; and it can indicate the degree of disease activity in rheumatoid arthritis and other inflammatory arthropathies.

In the normal knee joint there is less than $3 \mathrm{ml}$ of clear, pale yellow, highly viscous fluid. It contains less than 750 leucocytes $/ \mathrm{mm}^{3}$. The majority of cells are small lymphocytes, and the remainder are neutrophil polymorphs and mononuclear phagocytes.
In osteoarthrosis the fluid is usually increased in amount, but remains clear and often retains its high viscosity. The total and differential cell count seldom differs from the normal.

In rheumatoid arthritis the fluid is increased in amount, is cloudy due to increased cellularity, and has a reduced viscosity so that it is often watery in consistency. Clot may form due to its increased content of fibrogen. The total cell count is usually raised above 3000 leucocytes $/ \mathrm{mm}^{3}$, and may exceed 50000 leucocytes $/ \mathrm{mm}^{3}$. Neutrophil polymorphs comprise over $50 \%$ of the cells present in fluids having high cell counts associated with active arthritis, but may comprise less than $50 \%$ of the cells in fluids having low cell counts during inactive phases of the disease. The content of albumin, globulin, IgA, IgG, IgM, and complement in the fluid is increased.

During acute episodes of crystal synovitis the fluid may be purulent in appearance, but the total and differential cell count is largely dependent upon the number of crystals present. Crystals of sodium urate (gout) and calcium pyrophosphate dihydrate (pseudogout) are identified by their specific optical properties.

In septic arthritis the total cell count may be quite low in the early stages and thus may easily be confused with an inflammatory arthropathy or crystal arthritis. In all doubtful cases, the presence of microorganisms is investigated using appropriate special stains and by culture.

In posttraumatic effusions red blood cells may only be numerous for a short time after the traumatic episode has occurred. Red blood cells may also be numerous due to the aspiration procedure, acute synovitis of any cause, haemophilia, or villonodular synovitis. Breaching of the articular surfaces following trauma or bone necrosis may be revealed by the presence of numergus intracellular fat droplets following ithe uptake of bone marrow fat by synopal fluid leucocytes.

Platelet Aggregation with Ristocetin

JANE HUGHES, ELAINE WILSON, T. $\S$. BARROWCLIFFE, AND PENELOPE STABLEFORTH (The Royal Free Hospital, Londen) Following the reports of Howard क्ष Firkin (1971, 1973) that Ristocefin induced platelet aggregation was valuable diagnostic test for $\oplus_{0}$ Willebrand's disease (vWd), 30尺 płeviously diagnosed von Willebrand patients were reinvestigated. Standard in gations of blood coagulation and pettet function were carried out, as well a measurement of the factor VIII-rela롤 protein and Ristocetin-induced aggoegation. With a final Ristocetin concentration of $1.0 \mathrm{mg} / \mathrm{ml}$, aggregation of Willebrand platelet-rich plasma (PRP) reduced in all patients (less than $1 \bar{\Theta}^{\circ} \%$ aggregation compared with the control range of $30-70 \%$ ). In 29 out of 30 patients the findings supported the hypothesis that the von Willebrand factor is in the plasma, since Ristocetin-induced aggfegation of von Willebrand platelets fesuspended in normal (N) platelet peor plasma (PPP) was normal, while aggiegation of N-platelets in von Willebrand PPP was abnormal. The exception to this was patient 19, in whom mixily experiments indicated a platelet defect (see table).

Twenty-eight patients had reduced or absent levels of factor VIII related protein, as reported by Zimmermâh, Ratnoff, and Powell (1971).

Since the two patients with normal levels of VIII-related protein, cases 7 and 19 , had hereditary patterns c\&nsistent with an autosomal dominant inheritance, they were dissimilar from the cases of von Willebrand's disease whth 


\begin{tabular}{llll}
\hline & PRP & N Platelets/von Willbrand's PPP & von Willebrand's Platelets/N PPP \\
\hline $\begin{array}{l}\text { Case } 19 \\
\begin{array}{l}\text { All other } \\
\text { cases }\end{array}\end{array}$ & Abnormal & Normal & Abnormal \\
\hline
\end{tabular}

Table

normal VIII-related protein, as described by Holmberg and Nilsson (1973).

The three patients to whom cryoprecipitate was given all showed a postinfusion correction of Ristocetin aggregation, although the response of factor VIII and VIII-related protein varied in each case.

To the standard definition of classical von Willebrand's disease, it is now possible to add abnormal Ristocetin-induced aggregation and reduced or absent levels of factor-VIII-related protein. However, more variants of this disorder, two of which have been discussed in this paper, are likely to be described.

\section{References}

Holmberg, L., and Nilsson, I. (1973). New Engl. J. Med., 288, 595.

Howard, M., and Firkin, B. (1971). Thrombos. Diathes. Haemorrh. (Stuttg), 26, 362.

Howard, M., and Firkin, B. (1973). Blood, in press.

Zimmerman, T. S., Ratnoff, O. D., and Powell, A. E. (1971). J. clin. Invest., 50, 244.

Thrombotic Tendency and the Efficacy of Long-term Oral Anticoagulant Therapy as Demonstrated by Laboratory Tests

R. D. EASTHAM (Frenchay Hospital, Bristol) Following haemorrhage, trauma, surgery, or thrombosis, or in association with carcinoma, the plasma-activated partial thromboplastin clotting time (APTT) tends to be at the lower end of the normal range, or even below it. There is a direct correlation between the prothrombin ratio and the corresponding APTT in blood samples taken from patients treated with long-term oral anticoagulants. In patients treated with oral anticoagulants following venous thrombosis, at any given prothrombin ratio value, the corresponding APTT tends to be lower than in patients with mitral valve disease or following myocardial infarction, similarly treated. In an attempt to define this difference, 5088 results of prothrombin ratios and their corresponding APTT values obtained from 435 patients during 4564 months of anticoagulant therapy have been plotted on a grid.

It was found that there was a highly significant difference in the distribution of results from patients following myocardial infarction and those following venous thrombosis, whereas there was no significant difference in results from patients following myocardial infarction and those with mitral valve disease. Similarly, there was no significant difference between results from patients treated following venous thrombosis and those who had been treated following cerebrovascular damage. There was evidence of significant change in response to treatment after three months following postoperation venous thrombosis and after one year following so-called 'spontaneous' venous thrombosis, but there was no significant change in response in patients treated following myocardial infarction, during their first, second, or third years of treatment.

Quality Control Trials in the British System for Anticoagulant Control

L. POLLER, J. M. THOMSON, AND I. LECK (Withington Hospital, Manchester) The British system for anticoagulant control depends on an official national reagent, the British Comparative Thromboplastin (BCT) and a national system of reporting prothrombin time results, the British Corrected Ratio. There have been eight national quality control studies in Britain in the last two years involving nearly 300 hospitals.

The underlying assumption with the British reference scheme using the BCT was that all participating hospitals would obtain similar results using the national reagent and the recommended prothrombin time technique as described in the Association of Clinical Pathologists' Broadsheet. Quality control trials were designed to test this principle. Studies involved the use of both test thromboplastin reagents and lyophilized plasma samples. The following points emerged: there was considerable individual variation in results from hospital to hospital. Some hospitals tended to produce consistently higher ratios than others.

Three different methods of dealing with the problem appear to be required:(1) a programme of technical training to $\stackrel{2}{\overrightarrow{2}}$ focus attention on the technical variables 을 of the prothrombin time test; (2) the $\overrightarrow{\vec{F}}$ widespread provision of the standardized $\stackrel{\oplus}{\rightarrow}$ Manchester thromboplastin reagent for $\bar{C}$ use in all routine prothrombin time tests; (3) the regular and widespread provision of standardized lyophilized plasma quality control samples.

The British system for anticoagulant कै control based on the use of a national $\overrightarrow{0}$ thromboplastin reagent with a national $\overrightarrow{\vec{c}}$ system of reporting supported by national $\vec{\omega}$ quality control studies using lyophilized plasma preparations thus appears to offer $\frac{}{0}$ a model which many countries abroad is have begun to copy to solve their own national problems. Good progress has $N$ already been made in this direction in $\mathscr{\varrho}$ some Commonwealth countries and in South Africa.

A Comparison of Different Methods of $\rightarrow$ Detecting Mucin in Adenocarcinoma of $\mathbb{D}$ the Lung

A. KENNEDY AND P. D. BURGIN (Royal Infirmary, Sheffield) The correct classification of carcinomas of the lung is not $\vec{v}$ only of therapeutic and prognosec importance but is also considered to have epidemiological and aetiological signiff cance. Histological tests for mucin are essential in the classification of lung tumours but there is little information available about the influence of the method $\stackrel{D}{2}$ of detection used on the results obtained. $\overrightarrow{\vec{O}}$ Five established staining techniques were $\frac{}{3}$ tested using paraffin blocks from surgical $\vec{\supset}$ specimens of 81 human lung tumours diagnosed as adenocarcinoma, ie, tumours of WHO type III.

Mowry's alcian blue-PAS technique: gave the highest proportion of positives (93\%), slightly fewer $(90 \%)$ being ob-i tained by the PAS technique alone. Both these methods were influenced by the presence of cytoplasmic hyaline globules, $\supset$ structures which cannot be regarded as $D$ mucin. The stain recommended by the World Health Organization was also $\mathrm{N}$ influenced by the presence of hyaline $\Omega$ globules, was less frequently positive than $N$ the PAS techniques, and was considered N to have no special advantages. The $\sigma$ aldehyde fuchsin-alcian blue sequence was positive in only $83 \%$ of cases but $\stackrel{\circ}{\complement}$ provided some information about the $\mathbb{Q}$ type of mucin present. Southgate's? mucicarmine also detected mucin in $\square$ only $83 \%$ of cases.

It was concluded that the apparent 